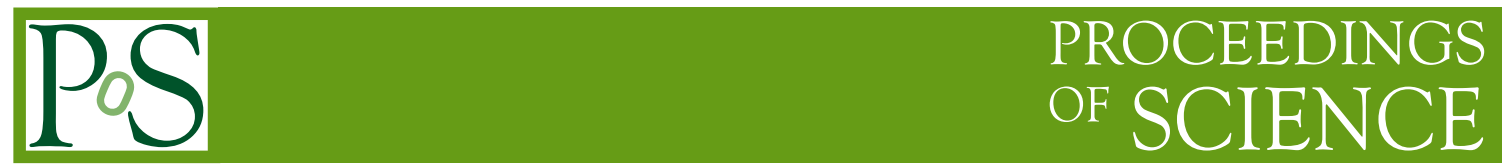

\title{
Current and future observations of the high-z IGM with the 21-cm line
}

\section{G. Bernardi*}

SKA SA, 3rd Floor, The Park, Park Road, Pinelands, 7405, South Africa;

Department of Physics and Electronics, Rhodes University, PO Box 94, Grahamstown, 6140,

South Africa

E-mail: gbernardieska.ac.za

Observations of the redshifted $21-\mathrm{cm}$ line can probe the evolution of the intergalactic medium even prior widespread reionization. In this contribution I briefly review the status of $21-\mathrm{cm}$ observations and what they start telling us about the evolution of the InterGalactic Medium at high redshift $(z>6)$.

XII Multifrequency Behaviour of High Energy Cosmic Sources Workshop 12-17 June, 2017

Palermo, Italy

${ }^{*}$ Speaker. 


\section{Introduction}

The redshifted 21-cm line is an ideal probe of cosmic reionization as its brightness $\delta T_{b}(z)$ directly depends upon the neutral fraction of the InterGalactic Medium (IGM) $x_{\mathrm{HI}}$ :

$$
\begin{gathered}
\delta T_{b}(z) \approx 27 x_{\mathrm{HI}}(z)\left(1-\frac{T_{\gamma}(z)}{T_{s}(z)}\right)(1+\delta(z))\left(\frac{H}{\mathrm{~d} v_{r} / \mathrm{d} r+H}\right) \\
\sqrt{\frac{1+z}{10} \frac{0.15}{\Omega_{\mathrm{M}} h^{2}}}\left(\frac{\Omega_{\mathrm{b}} h^{2}}{0.023}\right)\left(\frac{1-Y_{p}}{0.75}\right) \mathrm{mK},
\end{gathered}
$$

where $T_{\gamma}$ is the cosmic microwave background (CMB) temperature, $T_{s}$ is the spin temperature, $\delta$ is the fractional overdensity in baryons, $\mathrm{d} v_{r} / \mathrm{d} r$ is the peculiar velocity term, $\Omega_{\mathrm{M}}=0.315$ is the matter density, $h \equiv H /\left(100 \mathrm{~km} \mathrm{~s}^{-1} \mathrm{Mpc}^{-1}\right)=0.673$ is the normalized Hubble parameter and $\Omega_{\mathrm{b}}=0.049$ is the baryon density (Planck collaboration, 2015). Field (1959) showed that the resonant scattering of Ly $\alpha$ photons from $\mathrm{HI}$ atoms can quickly couple the spin temperature to the gas temperature $T_{k}$ (i.e. $\left.T_{s}=T_{k}\right)$ in the high redshift $(z>6)$ IGM. Before widespread reionization, therefore, the $21-\mathrm{cm}$ signal is a sensitive probe of the thermal history of the IGM.

Theoretical models identify different epochs in the evolution of the $21-\mathrm{cm}$ brightness temperature (i.e. Furlanetto 2006, Pritchard \& Loeb 2010, Mesinger, Greig \& Sobacchi 2016):

- $z_{\alpha}<z<z_{*}$ : the first stars are formed at $z_{*}$ and are expected to emit Ly $\alpha$ photons that will start driving the spin temperature to the gas temperature via resonant scattering (Wouthuysen 1952, Field 1959). Full coupling is achieved at $z_{\alpha}$;

- $z_{h}<z<z_{\alpha}$ : after Ly $\alpha$ coupling, sufficient radiation is released and starts heating the IGM. Most of the gas remains colder than the CMB apart from small pockets around the brightest sources: the colder the gas with respect to the $\mathrm{CMB}$, the brighter (in absorption) the $21-\mathrm{cm}$ signal

- $z_{s}<z<z_{h}$ : as heating continues, the gas temperature is eventually driven above the CMB temperature until saturation, i.e. $T_{s}=T_{k} \gg T_{\gamma}$, is reached at $z_{s}$. From this point onwards, the 21-cm emission is no longer a sensitive probe of the thermal state of the IGM;

- $z_{r}<z<z_{s}$ : after saturation, the ionization fraction raises to several percents due to the continued emission of ionizing photons from the first galaxies, marking the beginning of cosmic reionization $\left(z_{r}\right)$. Although most models make this assumptions, if gas heating is inefficient, saturation may happen after the ionization fraction start to significantly increase (i.e., $z_{s}<z<z_{r}$ ), leading to a "cold reionization", i.e. with $T_{k}<T_{\gamma}$ (Mesinger, Ferrara \& Spiegel 2013; Fialkov, Barkana \& Visbal 2014).

The sources of IGM heating remain rather uncertain even from a theoretical standpoint, but their main constituent is generally accepted to be X-ray emission from first stellar black holes (e.g., Pritchard \& Furlanetto 2007) or very hot interstellar medium (e.g., Pacucci et al. 2014), although Ly $\alpha$ radiation and shocks are contributing at a minor level (e.g., McQuinn \& O'Leary 2012). Observations of the $21-\mathrm{cm}$ emission before the spin temperature is saturated can, therefore, indirectly probe the presence and nature of X-ray sources in the early Universe. 


\section{Constraints on the IGM temperature from $21-\mathrm{cm}$ observations}

Observations of the redshifted $21-\mathrm{cm}$ are carried out using low frequency radio astronomical instrumentation: if they target the sky-averaged (global) signal they use a single dipole operating over a relatively large frequency range (i.e. Bowman \& Rogers 2010; Bernardi, McQuinn \& Greenhill 2015; Singh et al. 2015), whereas if they aim to measure fluctuations above the mean signal they require the angular resolution provided by interferometric arrays.

The presence of Galactic and extragalactic foregrounds that are a few orders of magnitude brighter than the 21-cm emission everywhere in the sky (e.g., Bernardi et al. 2009, 2010; Dillon et al. 2014; Parsons et al. 2014) is the greatest hurdle to detect the signal, however, recent observations with the Precision Array to Probe the Epoch of Reionization (PAPER, Parsons et al. 2010) have managed to derive upper limits to the $21-\mathrm{cm}$ signal at $z=8.4$ that provide a first evidence that the IGM was heated at $z=z_{*}>8.4$ (Ali et al. 2015; Pober et al. 2015; Greig, Mesinger \& Pober 2016; Figure 1).
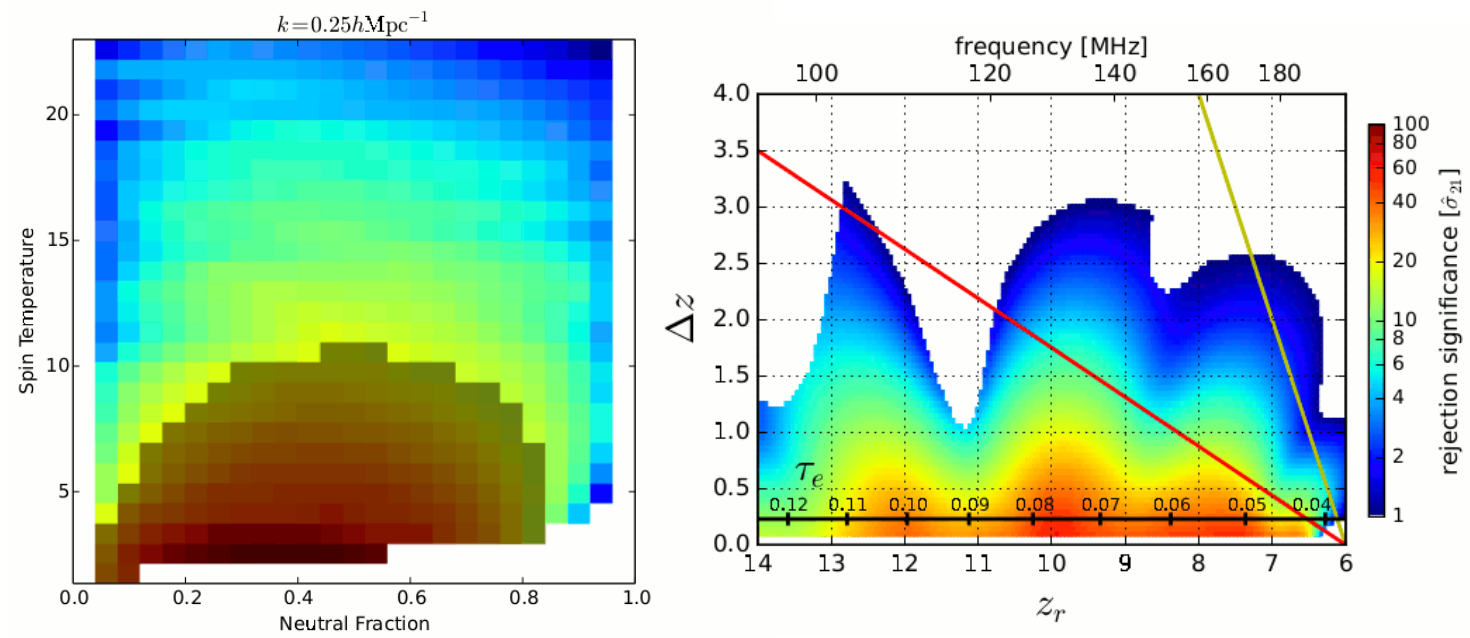

Figure 1: Left: constraints on the IGM spin temperature at $z=8.4$ (from Pober et al. 2015), assuming full copling between the gas and spin temperature, $T_{k}=T_{s}$. Right: constraints on the durationa and mid-point of reionization for "cold reionization" scenarios from global signal measurements (from Monsalve et al. 2017, see text for details).

As reionization is believed to be well underway at $z_{*}=8.4$, the $21-\mathrm{cm}$ brightness temperature depends on both the HI neutral fraction and the gas temperature (equation 1.1), under the 
assumption of full coupling between the gas and spin temperature. Given a certain neutral fraction, however, the magnitude of the $21-\mathrm{cm}$ brightness fluctuations is inversely proportional to the gas temperature, making the reasonable assumption that the fluctuation power is approximately constant over a wide range of modes (e.g., McQuinn et al. 2007). The PAPER fluctuation upper limits lead to a $T_{k} \gtrsim 6 \mathrm{~K}$ lower limit on the gas temperature at $z_{*}=8.4$. This value can be compared against the gas temperature expected in a "maximally cold" model, e.g. a model where the gas was never heated and the spin temperature was instantaneously coupled to the gas temperature when the first stars were formed (e.g., Bernardi et al. 2016; Monsalve et al. 2017). Although such model is somewhat unrealistic, it provides a lower bound to the gas temperature.

In a maximally cold model, the gas temperature at $z_{*}$ can be obtained by assuming adiabatic cooling - $T_{k} \propto(1+z)^{2}$ - from thermal decoupling between the CMB and the gas at redshift $z_{d} \approx 150$ (e.g., Furlanetto 2006):

$$
T_{k, c}\left(z_{*}\right)=T_{s}\left(z_{*}\right)=T_{\gamma, 0}\left(1+z_{d}\right)\left[\frac{1+z_{*}}{1+z_{d}}\right]^{2} \simeq 1.6 \mathrm{~K},
$$

(recalling that the CMB temperature today is $T_{\gamma, 0}=2.726 \mathrm{~K}$ ), which requires gas heating at $z>8.4$ in order not to violate the upper limits and implying that reionization may not be "completely cold".

Similar conclusions are drawn from observations of the global 21-cm signal in the $6<z<12$ range that set joint constraints on the duration and mid-point of reionization (when $x_{\mathrm{HI}}=0.5$ ) together with a $T_{b}>-100 \mathrm{mK}(2 \sigma)$ upper limit amplitude on the $21-\mathrm{cm}$ signal (Monsalve et al. 2017; Figure 1). For a maximally cold model, this constraint implies $T_{k}\left(z_{*}\right) \gtrsim 5 \mathrm{~K}$, again implying that reionization cannot be completely cold and the IGM must have been previously heated.

The presence of an heating X-ray background would be more readily inferred from observations at even higher redshift, where the IGM is essentially fully neutral and the $21-\mathrm{cm}$ signal only depends upon the gas temperature. Bernardi et al. (2016) presented a $-890 \mathrm{mK}$ upper limit on the amplitude of the $21-\mathrm{cm}$ absorption in the $13<z<27$ range, implying $T_{k} \gtrsim 3 \mathrm{~K}$. A maximally cold model, however, leads to $T_{k, c}(z=25) \sim 12 \mathrm{~K}$, therefore more sensitive observations are needed to place meaningful constraints on the IGM temperature prior reionization.

\section{Conclusions and future outlooks}

In this paper I have briefly reviewed current measurements of the temperature of the IGM at high redshift $(z>6)$ using $21-\mathrm{cm}$ line observations. Although in their early days, these observations already provide some evidence that the IGM must have been heated at $z \gtrsim 8.4$. Although the nature of the heating sources remains unconstrained at the current sensitivity levels, future observations may be able to probe the nature of X-ray sources in the early Universe, that are expected to provide the dominant heating contribution in most theoretical models.

\section{Acknowledgments}

This work is based on research supported by the National Research Foundation under grant 92725. Any opinion, finding and conclusion or recommendation expressed in this material is that of the author(s) and the NRF does not accept any liability in this regard. This work is based on 
the research supported in part by the National Research Foundation of South Africa (grant No. 103424).

\section{References}

[1] Ali, Z.S., Parsons, A.R., Zheng, H., et al. 2015, ApJ, 809, 61

[2] Bernardi, G., de Bruyn, A.G., Brentjens, M.A., et al. 2009, A\&A, 500, 965

[3] Bernardi, G., de Bruyn, A.G., Harker, G., et al. 2010, A\&A, 522, A67

[4] Bernardi G., McQuinn M. \& Greenhill L.J., 2015, ApJ, 799, 90

[5] Bernardi G. et al., 2016, MNRAS, 461, 2847

[6] Bowman J.D., Rogers A.E.E., 2010, Nature, 468, 796

[7] Dillon, J.S., Liu, A., Williams, C.L., et al. 2014, Phys. Rev. D, 89, 023002

[8] Field G.B., 1959, ApJ, 129, 536

[9] Fialkov A., Barkana R., Visbal E., 2014, Nature, 506, 197

[10] Furlanetto S.R., 2006, MNRAS, 371, 867

[11] Greig B., Mesinger A. \& Pober J.C., 2016, MNRAS, 455, 4295

[12] McQuinn M., Lidz A., Zahn O., Dutta S., Hernquist L. \& Zaldarriaga, M., 2007, MNRAS, 377, 1043

[13] McQuinn M. \& O’Leary R.M., 2012, ApJ, 760, 3

[14] Mesinger A., Ferrara A. \& Spiegel D.S., 2013, MNRAS, 431, 621

[15] Mesinger A., Greg B. \& Sobacchi E., 2016, MNRAS, 459, 2342

[16] Monsalve R.A., Rogers A.E.E., Bowman J.D. \& Mozdzen T. J., 2017, ApJ, 847, 64

[17] Pacucci F., Mesinger A., Mineo S., Ferrara A., 2014, MNRAS, 443, 678

[18] Parsons, A.R., Backer, D.C., Foster, G.S., et al. 2010, AJ, 139, 1468

[19] Parsons, A.R., Liu, A., Aguirre, J.E., et al. 2014, ApJ, 788, 106

[20] Planck Collaboration, 2015, 2016, A\&A, 594, A13

[21] Pober, J. C., Ali, Z. S., Parsons, A. R., et al. 2015, ApJ, 809, 62

[22] Pritchard J.R., Furlanetto S. R., 2007, MNRAS, 376, 1680

[23] Pritchard J.R., Loeb A., 2010, Phys. Rev. D, 82, 023006

[24] Singh S., Subrahmanyan R., Udaya Shankar N. \& Raghunathan A., 2015, ApJ, 815, 88

[25] Wouthuysen S.A., 1952, AJ, 57, 31 